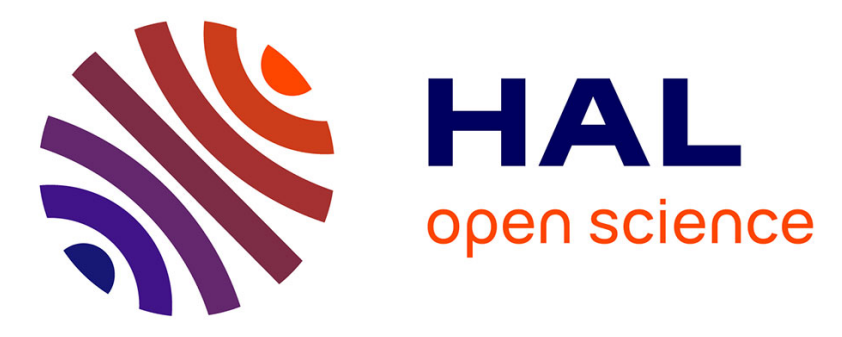

\title{
Solution-Processed Barium Titanate Nonlinear Woodpile Photonic Structures with Large Surface Areas
}

Viola Valentina Vogler-Neuling, Romolo Savo, David Pohl, Nicholas Hendricks, Lukas Lang, Maria Timofeeva, Barbara Schneider, Felix Ulrich Richter, Flavia Timpu, Serge Monneret, et al.

\section{To cite this version:}

Viola Valentina Vogler-Neuling, Romolo Savo, David Pohl, Nicholas Hendricks, Lukas Lang, et al.. Solution-Processed Barium Titanate Nonlinear Woodpile Photonic Structures with Large Surface Areas. physica status solidi (b), 2020, 257 (5), pp.1900755. 10.1002/pssb.201900755 . hal-03222989

\section{HAL Id: hal-03222989 \\ https://hal-amu.archives-ouvertes.fr/hal-03222989}

Submitted on 11 May 2021

HAL is a multi-disciplinary open access archive for the deposit and dissemination of scientific research documents, whether they are published or not. The documents may come from teaching and research institutions in France or abroad, or from public or private research centers.
L'archive ouverte pluridisciplinaire HAL, est destinée au dépôt et à la diffusion de documents scientifiques de niveau recherche, publiés ou non, émanant des établissements d'enseignement et de recherche français ou étrangers, des laboratoires publics ou privés.

\section{(ㄷ)(1) $\$$}

Distributed under a Creative Commons Attribution - NonCommerciall 4.0 International 


\title{
Solution-Processed Barium Titanate Nonlinear Woodpile Photonic Structures with Large Surface Areas
}

\author{
Viola Valentina Vogler-Neuling, Romolo Savo, David Pohl, Nicholas R. Hendricks, \\ Lukas Lang, Maria Timofeeva, Barbara Schneider, Felix Ulrich Richter, Flavia Timpu, \\ Serge Monneret, Fabian Starsich, and Rachel Grange*
}

Three dimensional (3D) nonlinear photonic crystals are one of the potential candidates for enabling photonic circuits. Most of the current top-down fabrication approaches for such crystals are time consuming and suffer from small surface areas and limitations to specific substrates. Herein, a bottom-up fabrication technique (UV-soft nanoimprint lithography) together with solution processing of barium titanate $\left(\mathrm{BaTiO}_{3}\right)$ nanoparticles is used to create large-scale 3D nonlinear woodpile photonic crystals. Opposed to other 3D photonic crystals, the woodpile structure can be fabricated layer by layer. This property makes it suitable for roll-to-roll fabrication, which can be easily adapted in industry. The prepared photonic crystals with up to eight layers have surface areas of $1.0 \mathrm{~cm}^{2}$. The linear and nonlinear optical behavior of these 3D woodpile structures is characterized. Their transmission stop band lies around $757 \mathrm{~nm}$ and a relative linear minimum transmission of $48.4 \%$ for a four-layer sample is obtained. Images of diffraction patterns together with polarization-dependent second harmonic generation measurements prove a cubic photonic crystal structure in the linear and nonlinear regime. In addition to second-order nonlinearity, $\mathrm{BaTiO}_{3}$ exhibits electro-optic, elasto-optic, and thermo-optic effects. Therefore, it is especially suited for future active photonic crystal applications.

data at the speed of light and by offering a low-power and high-bandwidth alternative to semiconductor materials. ${ }^{[1]}$ To create densely integrated 3D photonic circuits, structures capable of confining and routing light in all spatial directions need to be developed. Photonic crystals (PhCs), structures with a periodic modulation of the dielectric function, are key elements to enable such a control, due to their wavelength-specific transmission (photonic bandgap) and efficient mode confinement in the presence of designed defects. ${ }^{[2]}$ Nonlinear PhCs (NPhCs) are 3D PhCs made up of an optically nonlinear material. ${ }^{[3]}$ They have an increased functionality for integrated photonics compared with their linear counterparts as the nonlinear dielectric medium has an intrinsic ultrafast response to optical fields enabling alloptical high-speed operability. ${ }^{[3]} \mathrm{NPhCs}$ can be a platform to realize optical diodes, transistors, switches, and discrete soliton networks. ${ }^{[4]}$

However, such systems are still rare due

\section{Introduction}

The amount of data created every day requires new solutions for its storage and processing. The field of photonics offers the possibility to overcome these limitations by processing to their expensive and time-consuming fabrication processes resulting in structures of only small surface areas typically in the $\mu \mathrm{m}^{2}$ range. So far, a naturally grown $3 \mathrm{D} \mathrm{NPhC}$ in a $\mathrm{Ba}_{0.77} \mathrm{Ca}_{0.23} \mathrm{TiO}_{3}$ crystal was fabricated by the Czochralski method. ${ }^{[5]}$
V. V. Vogler-Neuling, Dr. R. Savo, D. Pohl, Dr. N. R. Hendricks, L. Lang, Dr. M. Timofeeva, B. Schneider, F. U. Richter, Dr. F. Timpu,

Prof. R. Grange

ETH Zurich

Department of Physics

Institute for Quantum Electronics

Optical Nanomaterial Group

Auguste-Piccard Hof 1, CH-8093 Zurich, Switzerland

E-mail: grange@phys.ethz.ch

The ORCID identification number(s) for the author(s) of this article can be found under https://doi.org/10.1002/pssb.201900755.

(C) 2020 The Authors. Published by WILEY-VCH Verlag GmbH \& Co. KGaA, Weinheim. This is an open access article under the terms of the Creative Commons Attribution-NonCommercial License, which permits use, distribution and reproduction in any medium, provided the original work is properly cited and is not used for commercial purposes.

\author{
Dr. S. Monneret \\ Aix Marseille Univ \\ CNRS \\ Ecole Centrale Marseille \\ Institut Fresnel \\ 13397 Marseille, France \\ Dr. F. Starsich \\ ETH Zurich \\ Department of Mechanical and Process Engineering \\ Institute of Process Engineering \\ Particle Technology Laboratory \\ Sonneggstrasse 3, CH-8092 Zurich, Switzerland
}

DOI: 10.1002/pssb.201900755 
In the same material, a 3D NPhC was fabricated using an ultrafast light domain inversion approach. ${ }^{[6]}$ Recently, a quasi-secondharmonic 3D NPhC in the mid-infrared region made of a bilayered structure on periodically poled lithium tantalate staggered in the depth direction was demonstrated and a 3D NPhC in $\mathrm{LiNbO}_{3}$ prepared by laser irradiation was shown as well. ${ }^{[7,8]}$

In these pioneering works, the focus is on the investigation of the quasiphase matching behavior of the NPhCs and on the diffraction patterns along different $\mathrm{PhC}$ axes. No attention was yet turned to the 3D NPhC's linear and nonlinear bandgap comparable with the works for $1 \mathrm{D}$ and 2D NPhCs. ${ }^{[9-11]}$ Furthermore, most of these structures depend on specific substrates and follow a top-down approach, which makes a scalable production challenging.

To create commercially available 3D NPhCs, first, an appropriate nonlinear optical material has to be found that can be integrated in a 3D PhC structure, which can be realized with a scalable and low-cost bottom-up fabrication technique.

We choose barium titanate $\left(\mathrm{BaTiO}_{3}\right)$, which shows one of the highest $\chi^{(2)}$ susceptibilities $\left(\langle d\rangle=14.1 \mathrm{pm} \mathrm{V}^{-1}\right.$ for bulk) among metal oxides and is, therefore, an appropriate choice for the realization of 3D NPhCs. ${ }^{[12]}$

This material is structured in woodpile PhCs that have already shown their high potential for a large amount of applications in various materials, wavelength ranges, and with different fabrication techniques. ${ }^{[13-17]}$ Unlike other 3D PhC structures, the woodpile structure allows a layer-by-layer production.

Finally, UV-soft nanoimprint lithography (UV-SNIL) combined with nanoparticle (NP) solution processing is a promising bottom-up fabrication technique to scale the size of PhCs up to large surface areas in the order of $\mathrm{cm}^{2}$ or bigger and is capable of roll-to-roll fabrication. ${ }^{[16,18]}$

In our work, we first extend the UV-SNIL technique to $\mathrm{BaTiO}_{3}$ NPs obtaining a scalable and inexpensive fabrication method for 3D NPhC. ${ }^{[19]}$ The fabricated and analyzed $\mathrm{BaTiO}_{3} 3 \mathrm{D}$ woodpile structures, with one to eight layers, are $\mathrm{PhCs}$ out of a nonlinear material with large surface areas of the order of $1.0 \mathrm{~cm}^{2}$. We observe a linear stop band at $757 \mathrm{~nm}$, providing evidence of coherent wave propagation and interference stemming from the PhC structure. The minimum relative linear optical transmission is achieved for a four-layer sample which yields $48.4 \%$ for $757 \mathrm{~nm}$. Second harmonic generation (SHG) polar measurements show that the $\mathrm{PhC}$ structure is cubic in the SHG regime.

\section{UV-SNIL and Solution Processing}

A silicon ( $\mathrm{Si}$ ) master mold has been realized by standard nanofabrication techniques (electron-beam lithography, development, and deep-reactive ion etching) from which a polydimethylsiloxane (PDMS) negative is taken. We stress that the Si master mold has to be fabricated only once and that a large number of negatives, called daughter molds, can be taken. This makes UV-SNIL a much less expensive technique compared with typical top-down approaches.

For the fabrication of the woodpiles, we first spin coat a 50:50 wt\% $\mathrm{BaTiO}_{3} \mathrm{NP} /$ titanium dioxide $\left(\mathrm{TiO}_{2}\right)$ sol-gel precursor solution onto a glass substrate (Figure 1a1 and Supporting Infor-

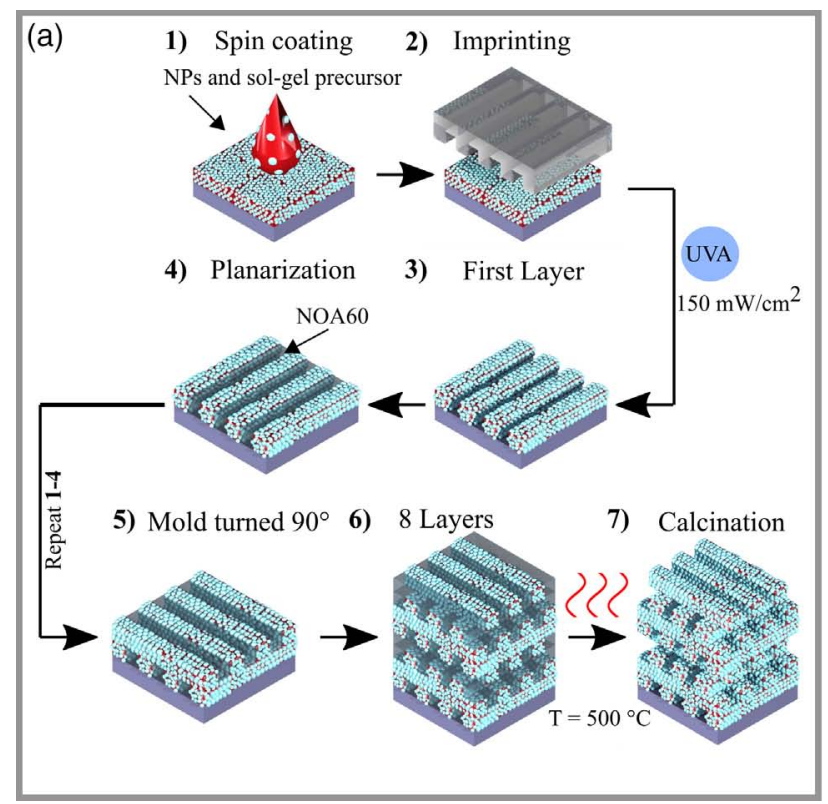

(b)

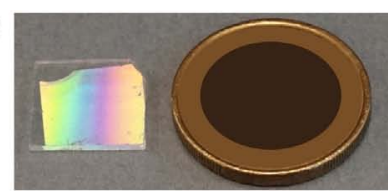

(c)

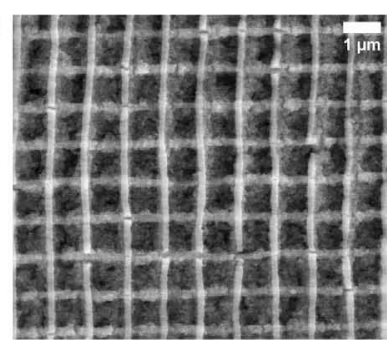

(d)

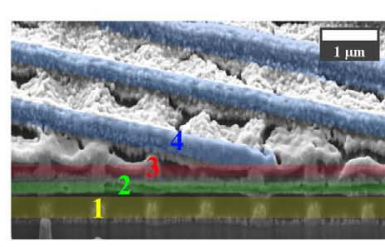

Figure 1. a) Fabrication process flow: 1) spin coating of NP/sol-gel precursor solution on a glass substrate; 2) imprinting with PDMS mold; 3) UV-light curing and removal of PDMS mask; 4) spin coating of the NOA60 planarization layers; 5) repeating steps (1)-(4) with mold orientation changed by $90^{\circ}$ with respect to the preceding layer orientation; 6) stack of eight woodpile layers; 7) removal of residual layers by calcination at $500^{\circ} \mathrm{C}$. b) Picture of the first layer of a photonic crystal on a glass substrate next to a chip that has the size of a one-Euro coin showing the typical surface area of $1.0 \mathrm{~cm}^{2}$. c) $\mathrm{SEM}$ topview image of an eight-layer sample ( $10 \mathrm{kV}$, secondary electrons in low vacuum mode at $70 \mathrm{~Pa})$. d) SEM image of a focused ion beam cut through a fourlayer woodpile sample showing the cross-section of the structures (SEM: $3 \mathrm{kV}$, FIB: $30 \mathrm{kV}: 10 \mathrm{pA}$ ). The numbers one (yellow) to four (blue) are labeling the layers, which are colorized in the corresponding color for better visibility. 
mation). The $\mathrm{BaTiO}_{3} \mathrm{NPs}$ have a mean diameter of (42 \pm 1$) \mathrm{nm}$ determined by scanning electron microscopy (SEM) analysis and a tetragonal crystal phase confirmed by powder X-ray diffraction measurements (Figure S1 and S2, Supporting Information). ${ }^{[20]}$ This NP size and crystal phase are known from previous studies to exhibit bulk second harmonic ( $\mathrm{SH}$ ) signals. ${ }^{[21]}$ The NP size is chosen as a compromise between having small particles for a good filling of the molds and large enough ones to exhibit bulk SH signals. In the second step, we place the PDMS mold on the coated substrate (Figure 1a2). Due to capillary forces, the PDMS mold fills with the NP/sol-gel precursor solution. In this position, we irradiate sample and mold with an UV flood lamp at $150 \mathrm{~mW} \mathrm{~cm}^{-2}$ for $180 \mathrm{~s}$ to crosslink the sol-gel precursor. After removal of the mold (Figure 1a3), two photoresist planarization layers (first $15 \mathrm{wt} \%$ NOA60 for coarse planarization and second $5 \mathrm{wt} \%$ NOA60 for fine planarization) are spin coated and exposed to UV light one after the other (Figure 1a4). We fabricate the consecutive layers in the same manner, each of them turned $90^{\circ}$ with respect to the preceding one (Figure 1a1-4). Finally, the samples are calcined at $500{ }^{\circ} \mathrm{C}$ to remove all organic residues especially the planarization layers to make the woodpile freestanding (Figure 1a7).

One typical layer of the cross-linked structure $\left(\mathrm{BaTiO}_{3}\right.$ NPs and $\mathrm{TiO}_{2}$ matrix) has a pitch of $1.002 \pm 0.031 \mu \mathrm{m}$, a height of $260 \pm 9 \mathrm{~nm}$, and a rod width of $506 \pm 31 \mathrm{~nm}$ determined with atomic force microscopy (AFM) and SEM (Figure 1c and S3a, Supporting Information). Figure $1 \mathrm{~b}$ shows a regular and homogeneous structure that demonstrates the ability of our fabrication method to produce large surface areas of up to $1.0 \mathrm{~cm}^{2}$. If the spin-coated film is not structured, the thin film of the composite appears transparent. The refractive index of the composite structure is measured with a combination of reflectometry and a phase-contrast camera and yields 1.77 at $800 \mathrm{~nm}$ (Figure S4, Supporting Information). ${ }^{[22]}$ Figure $1 \mathrm{c}$ shows a top view of an eight-layer sample and Figure 1d, a focus ion beam (FIB) cut of four layers of a four-layer sample. A regular structure is, therefore, also present on a microscopic scale. Small defects such as cracks in the rods, rotation misalignments in the stacking geometry, slight bendings of the rods, unsharp edges due to the NP size, and NP residuals are identified. They are increasing with an increasing amount of layers. Residual NPs have two major contributions on the optical behavior of the device. First, they can influence the bandgap position and depth because the geometry of the structure can be slightly varied. We investigated these effects (i.e., changes in period, width, height, and angles between layers) numerically. Simulations show that variations of the period influence the bandgap position most (Figure S6c, Supporting Information). Experimentally, the mask defines the period and residual NPs have a minimal impact on the period. Due to their distribution over the structure, residual NPs behave as defects and mainly attenuate the optical performance of the device, i.e., the depth of the bandgap. Second, residual particles that form clusters of sizes comparable with the wavelength introduce scattering and further attenuate the $\mathrm{PhC}$ behavior. Minimization of the amount of residuals would be possible by optimizing the volume of the solution for the spin coating and by introducing a soluble photoresist as a residual layer, which can be stripped away together with the residual NPs.

\section{Linear Optical Measurements}

To investigate the PhC structure, images of the diffraction patterns of one, two, and four layers of the woodpile PhC structure far away from the sample are taken while illuminating the whole samples $\left(1.0 \mathrm{~cm}^{2}\right)$ with a collimated white light halogen source (Figure $2 \mathrm{a}-\mathrm{c}$ ). For the one-layer sample, the orientation of the diffraction pattern is perpendicular to the orientation of the rods, as in diffraction gratings, and shows the capability to separate different wavelengths. For the two- and four-layer samples, the diffraction patterns reveal the one of the cubic $\mathrm{PhC}$ structures. ${ }^{[23]}$ For four layers (Figure 2c), a white underlying haze gets visible. This is the onset of multiple scattering due to the defects. We can conclude that the fabrication technique was successful and that the $\mathrm{PhC}$ structure is present in three dimensions.

To characterize the linear bandgap, we measured the transmission spectra of the structure for several incident polarizations with a spot diameter of $3 \mathrm{~mm}$ by collecting the zeroth order of the diffraction patterns via a fiber collimator guiding the light to a spectrometer. Figure $2 \mathrm{~d}$ shows the measured spectra for a single layer, corresponding to a grating of equidistant rods, where the $0^{\circ}$ polarization is perpendicular and the $90^{\circ}$ polarization is parallel to the rod orientation, respectively. We observe a clear dependence of the transmission on the incident polarization, with a difference in transmission between the orthogonal and parallel states of $21 \%$. For a polarization state of $90^{\circ}$, a minimum in transmission is appearing at a wavelength of $747 \mathrm{~nm}$, revealing the onset of a stop band.

Figure 2e shows the transmission spectra of woodpiles with an increasing amount of layers, from one to four layers, which are compared with the spectrum of an unpatterned thin film (TF 1)of the same composite with a thickness of $103 \mathrm{~nm}$. For each of the layers, two polarization states (\| and $\perp$ to the first layer of the samples) are shown. The absolute transmission is decreasing with an increasing amount of layers, as well as the difference in transmission between the two polarization states. A decline in transmission spectra to shorter wavelengths characterizes all woodpile structures, which can be attributed to incoherent scattering on the structure defects. ${ }^{[2,25]}$ Moreover, this decreasing linear trend is consistent with the extinction provided by a polydispersed ensemble of scatterers in a moderate multiple scattering regime. ${ }^{[26]}$ The barium titanate NPs of the composite are indeed too small (around $50 \mathrm{~nm}$ ) and too dense (around 70\% filling fraction) to generate this scattering, differently they provide an effective-medium optical response, as it is evident in the flatter transmission spectrum of the unpatterned film. The transmission stop band in the woodpile structures appears for all numbers of layers. However, from five layers onward, the scattering of the defects was so strong that we could not measure any features in the transmission.

To account for the two physical effects in the measurements (PhC behavior and multiple scattering from defects), we fitted a linear function to the data $\left(T_{\text {L.F. }}\right)$ (Figure $S 5 b$, Supporting Information), excluding the data between 700 and $800 \mathrm{~nm}$, and divided the transmission of the sample $\left(T_{\mathrm{S}}\right)$ by it (Figure $2 \mathrm{f}$, g). ${ }^{[25]}$ This normalization removes the multiple scattering component from the data and makes the $\mathrm{PhC}$ behavior clearer. 

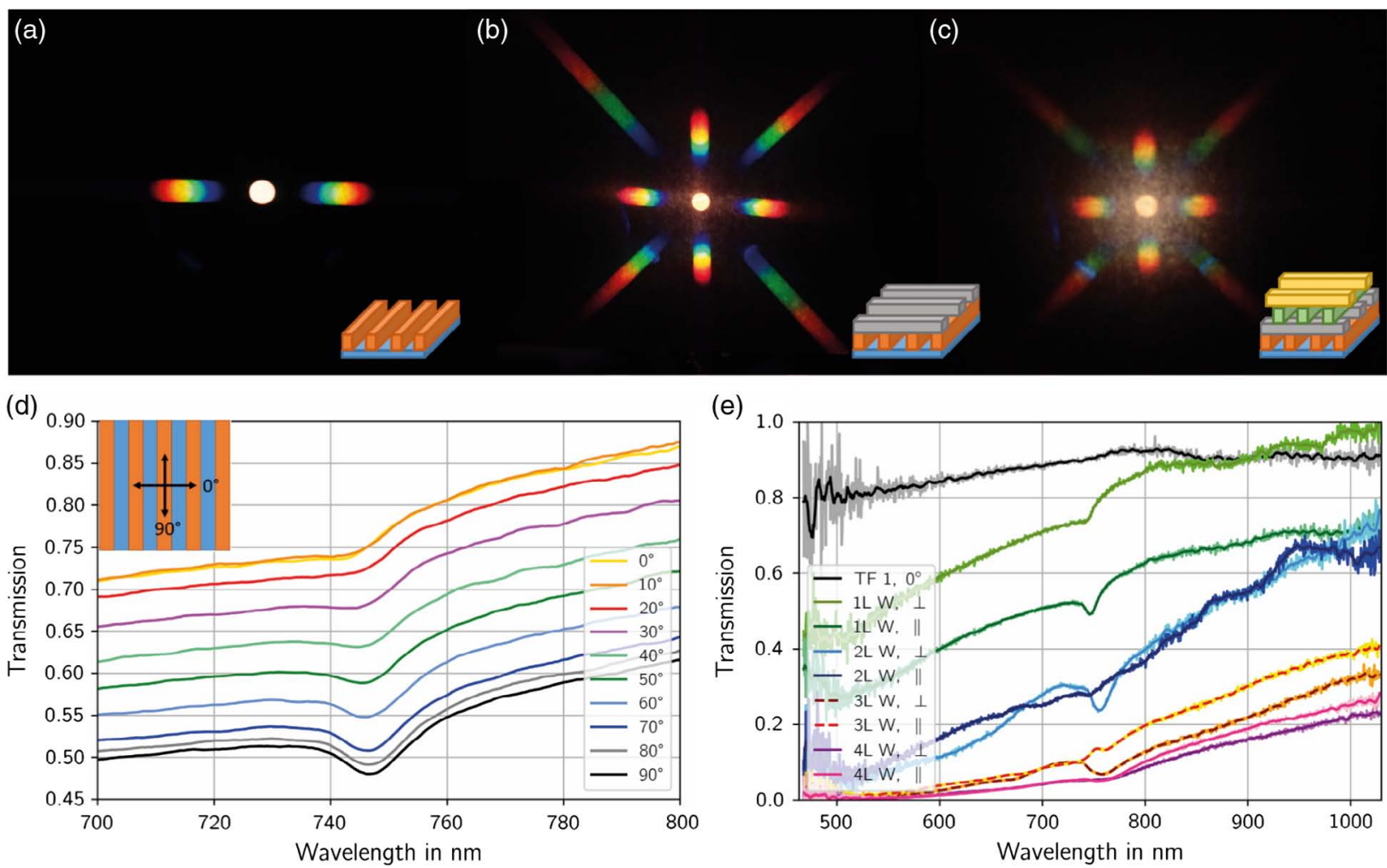

(e)
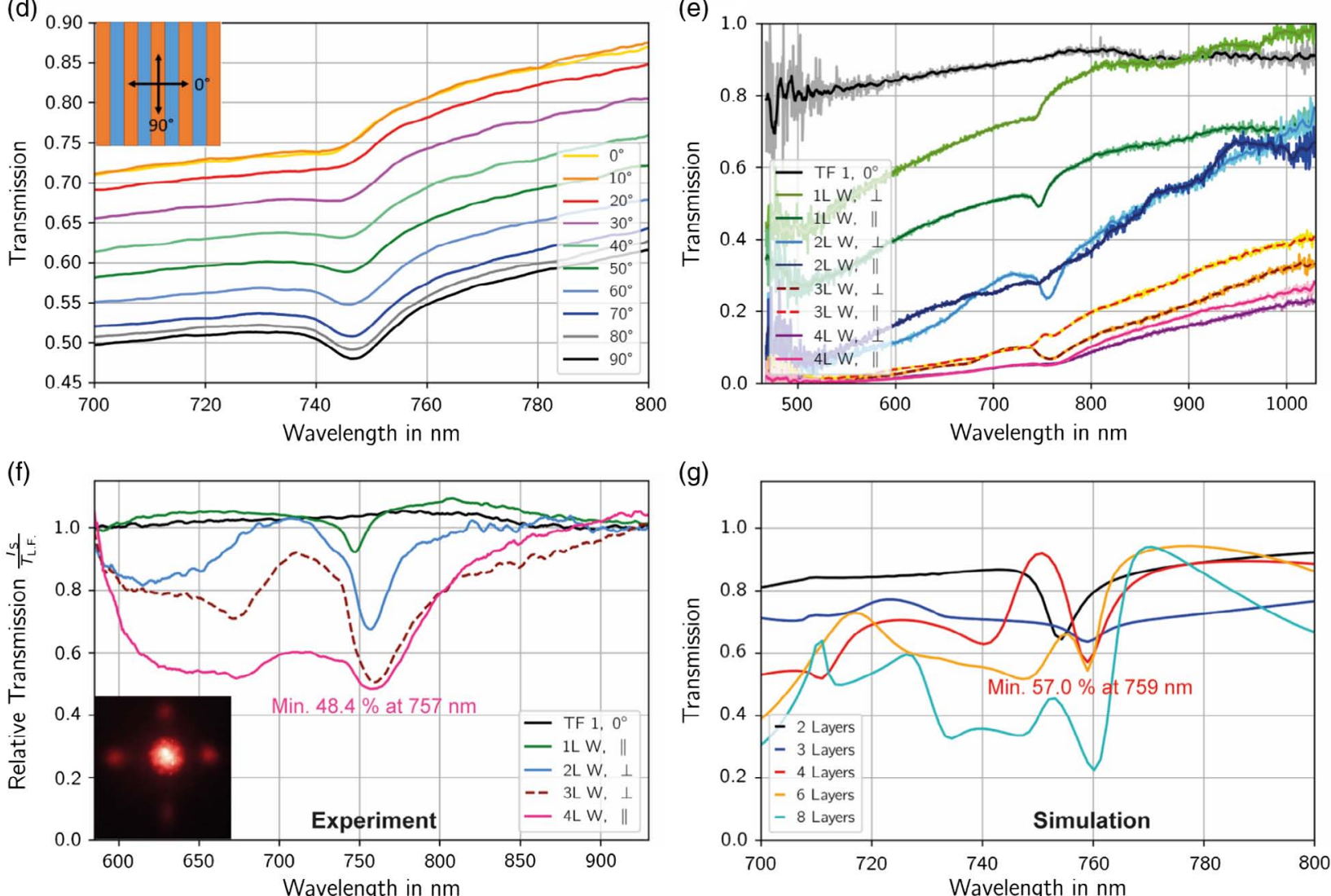

Figure 2. a-c) Images of diffraction patterns of a) one, b) two, and c) four layers. The diffraction pattern for the first layer is perpendicular to the orientation of the rods. For two and four layers, the obtained diffraction patterns are typical for a cubic photonic crystal structure. d) Polarizationdependent $\left(0^{\circ}-90^{\circ}\right)$ transmission data for the first layer where $0^{\circ}$ is defined perpendicular to the rod orientation and $90^{\circ}$ parallel, respectively. The absolute change in transmission between $0^{\circ}$ and $90^{\circ}$ is about $21 \%$. The feature at $747 \mathrm{~nm}$ is most pronounced for $90^{\circ}$ incident polarization. e) Layer-dependent transmission data for an unpatterned thin film (TF1) and one ( $1 \mathrm{~L} \mathrm{~W})$, two $(2 \mathrm{~L} \mathrm{~W})$, three ( $3 \mathrm{~L} \mathrm{~W})$, and four (4L W) layers of the woodpile structure for two incident polarizations $\left(0^{\circ}\right.$ and $\left.90^{\circ}\right)$, which is defined with respect to the first layer. The difference between the polarization states is decreasing with an increasing amount of layers. The absolute transmission value is decreasing with increasing layers due to scattering from defects. f) Close-up of the same measurements as in part (e) where we divided the transmission of the samples by the linear fit through the data. For a four-layer sample $\left(90^{\circ}\right)$, we achieved a minimal linear transmission of $48.4 \%$ at $757 \mathrm{~nm}$. Inset in part $(f)$ shows diffraction pattern for a four-layer woodpile with a superluminescent diode (SLED) of a center wavelength of $780 \mathrm{~nm}$. g) FDTD simulations for two to four layers with the experimental fabrication parameters. A transmission stop band is present at around $760 \mathrm{~nm}$ that corresponds to the experimental data in position and shape.

A minimum in transmission becomes pronounced at around $750 \mathrm{~nm}$. From three layers onward, this feature arises in both polarization states. The lowest relative transmission value of $48.4 \%$ was reached for the four-layer woodpile sample at a wavelength of $757 \mathrm{~nm}$.

\subsection{Finite-Difference Time-Domain Simulations of the Transmission Spectra}

According to the simulations (Figure $2 \mathrm{~g}$ and S6, Supporting Information) and previous work, ${ }^{[15]}$ the bandgap of the $3 \mathrm{D}$ 
photonic woodpile structure considered here is expected to be around $950 \mathrm{~nm}$ by design. To understand the origin of the transmission minimum observed at $757 \mathrm{~nm}$, finite-difference time-domain (FDTD) simulations were run on these structures. We simulated two to eight layers with different stacking geometry, $\mathrm{ABAB}$ or $\mathrm{ABCD}$, of a $3 \mathrm{D}$ woodpile $\mathrm{PhC}$ (cf., Figure S6a,b, Supporting Information) because of alignment imperfections we are between those two arrangements of the layers. For the parameters, we take the experimental mean values (period: $1.002 \mu \mathrm{m}$, height: $260 \mathrm{~nm}$, width $506 \mathrm{~nm}$, and effective refractive index: 1.775$)$ and the whole structure is placed on a glass substrate. The corresponding simulated transmission spectra are shown in Figure $2 \mathrm{~g}$ and S6, Supporting Information, and show two main features. One at around $760 \mathrm{~nm}$ (Figure 2g), which has a sharp shape and appears already from two layers onward and the other one around $950 \mathrm{~nm}$ (Figure S6, Supporting Information), which has a broader profile and only starts to be clearly seen from two or four layers onward (after one unit cell). The simulated feature around $759 \mathrm{~nm}$ matches very well the experimental case observed at $757 \mathrm{~nm}$, both in position and in depth with a reduced transmission of $57.0 \%$ and $48.4 \%$, respectively (cf., Figure $2 \mathrm{f}, \mathrm{g}$ ).

Simulations confirm that the transmission minimum at $757 \mathrm{~nm}$ found in experiments is not the PhC bandgap but a very pronounced side stop band, a feature that is expected in the high energy regime $(a / \lambda<1) .{ }^{[27]}$ This is consistent with the diffraction pattern around the stop band at a center wavelength of $780 \mathrm{~nm}$ (Figure 2f, inset), which should not be visible in the full photonic bandgap regime $(a / \lambda=1) .{ }^{[27]}$ Second, our simulations (Figure S6, Supporting Information) show that the bandgap around $950 \mathrm{~nm}$ is just arising with several layers and only shows pronounced features if at least one unit cell (four layers) is present. Furthermore, this behavior is expected to be valid for a higher order bandgap as well, i.e., it should not be visible from the first layer onward, as we experimentally observe it. This indicates that the feature at $757 \mathrm{~nm}$ is indeed not a higher order bandgap, but rather a side stop band, as described previously. Although we cannot observe the PhC bandgap because of the dominant structural imperfections for more than four layers, the emergence of this side stop band is physically very important as it gives evidence of coherent wave propagation and interference due to the woodpile PhC structure.

\section{Nonlinear Optical Measurements}

Second-order ( $\mathrm{SH})$ nonlinear optical signals are measured with a nonscanning transmission optical microscope. ${ }^{[28,29]}$ SHG power dependence measurements (cf., Figure $3 \mathrm{~g}$ and S7, Supporting Information) yield a quadratic dependence and prove that our signals are indeed SHG signals. To investigate the radiation behavior of the woodpile structures, we measured the SHG polarization response at wavelength steps of $100 \mathrm{~nm}$ in the fundamental wavelength. For the polarization measurements, a half-wave plate was rotated from $0^{\circ}$ to $180^{\circ}$ in steps of $2^{\circ}$ (cf., Figure 3a-e). All measurements were compared with the SHG from an unpatterned thin film of the same material. The shape of the SHG polarization response of the first layer of the woodpile PhC is dipolar (cf., Figure $3 \mathrm{~b}$ ). The long axis of the dipole is perpendicular to the orientation of the rods (cf., Figure 3c). A quadrupolar SHG response (cf., Figure 3e) is measured for two woodpile layers, suggesting that the two SHG polarization responses of the individual layers are summing up. Similarly, the SHG polarization response of the eight-layer woodpile sample corresponds to the sum of all layers and is again quadrupolar (cf., Figure 3e). It is known that by varying the PhC structure, we can manipulate the SHG radiation direction. ${ }^{[30]}$ Importantly, the observed SHG polarization patterns prove that the cubic $\mathrm{PhC}$ structure is also present in its nonlinear regime. As expected, the thin film of the same material (TF 1) shows a simple circular SHG polarization response, resulting solely from the average SHG of randomly oriented NPs in the composite (cf., Figure 3a).

To investigate the NPhC bandgap, we performed spectral SHG measurements. In the laser range of the femtosecond Ti:sapphire oscillator $(760-1060 \mathrm{~nm})$, we measured for all the samples at three different positions to average over imperfections in the structures. In the optical parametric oscillator (OPO) range (1060 to $1600 \mathrm{~nm})$, we determined the SHG spectra for three samples (TF 1, 1L W, and 8L W) at one position (Figure 3f). Above $1450 \mathrm{~nm}$, our sample exhibited a very strong third harmonic generation (THG) signal from the $\mathrm{TiO}_{2}$ matrix. This is in agreement with previously observed high THG signals of $\mathrm{TiO}_{2}$ in the anatase phase in thin films. ${ }^{[31]}$ This THG signal screened the SHG signal from $\mathrm{BaTiO}_{3}$ and limited the measurement range to $1400 \mathrm{~nm}$. This is why we could not directly probe the nonlinear transmission stop band, which was expected to arise at a fundamental wavelength of $1514 \mathrm{~nm}$ (twice the $757 \mathrm{~nm}$ ).

In the accessible wavelength range of $780-1400 \mathrm{~nm}$, we measured the squared effective $\chi^{(2)}$-tensor, which is an average value over the randomly oriented crystal orientations of the single NPs, of the woodpile PhCs (cf., Figure $3 f$ and Supporting Information). The highest measured value of the effective $\chi^{(2)}$. tensor is $1.15 \times 10^{-3} \mathrm{pm} \mathrm{V}^{-1}$ for the eight-layer woodpile sample at $800 \mathrm{~nm}$ (cf., Supporting Information for calculation of effective $\chi^{(2)}$-tensor), which corresponds to a conversion efficiency $(\gamma)$ of $9.5 \times 10^{-10}$ (Figure 3f). We expect that by sintering of the NPs and the induced crystal growth, this value can be enhanced in the future, as the SHG emission scales with the volume of the crystal.

One observes an increase in the effective $\chi^{(2)}$ from 700 to $400 \mathrm{~nm}$ second harmonic wavelength and a decrease in the effective $\chi^{(2)}$ below $400 \mathrm{~nm}$. An increasing value of $\chi^{(2)}$ close to the bandgap of a material is known from semiconductors and the bandgap of $\mathrm{BaTiO}_{3}$ is around $380 \mathrm{~nm} \cdot{ }^{[32-34]}$ We can reasonably assume that a similar effect is present here for $\mathrm{BaTiO}_{3}$, even though the $\chi^{(2)}$ behavior close to the bandgap of metal oxides has not yet been studied. Contributions from internal resonances inside the NPs can be neglected due to their small size. Furthermore, the bandgap position of $\mathrm{BaTiO}_{3}$ NPs could be slightly shifted due to their internal structure. This measurement technique could be an appropriate way to study the bandgap position of second harmonic NPs.

\section{Conclusion and Outlook}

In this work, we fabricated woodpile PhCs with one to eight layers by UV-SNIL, realizing large surface areas of PhCs in 
(a)

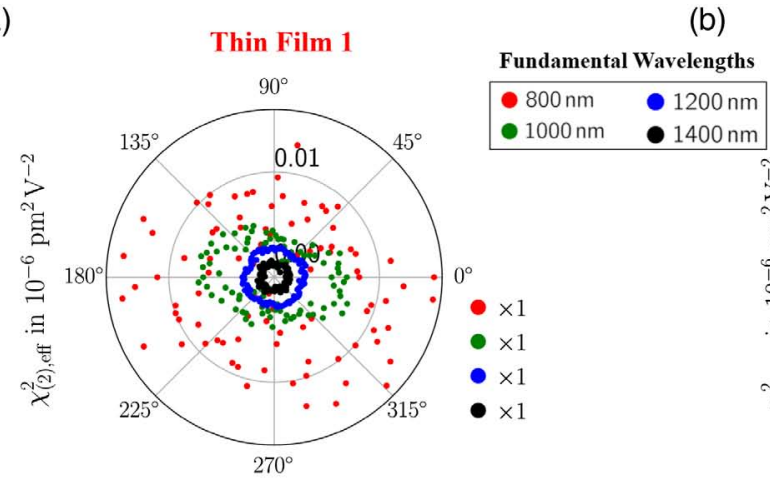

(c)

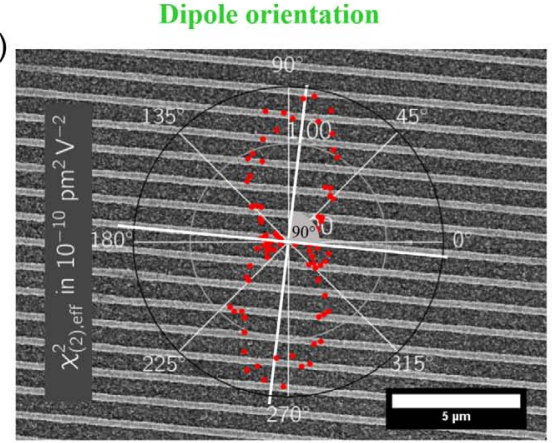

(e)

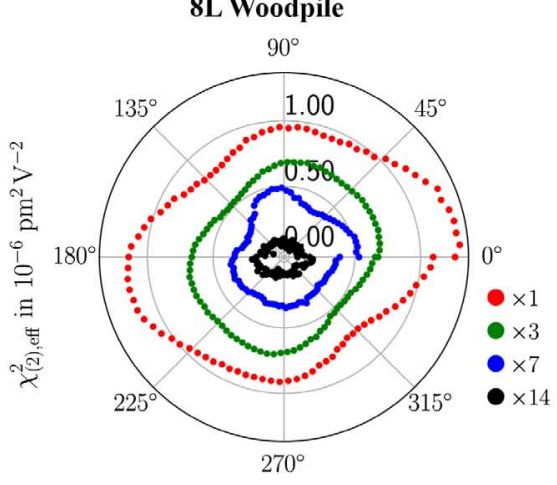

(b)

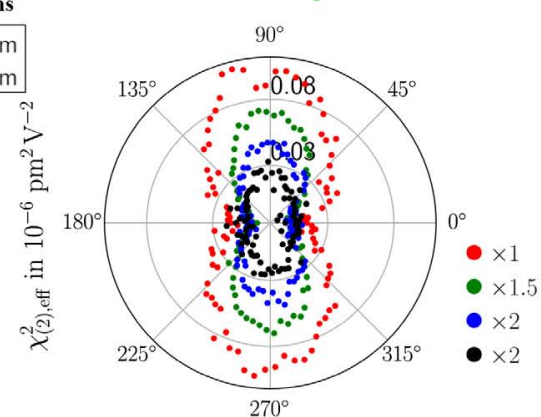

(d)

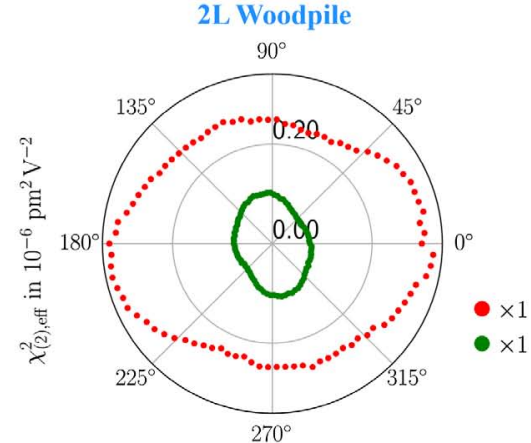

Second Harmonic Wavelength in $\mathrm{nm}$

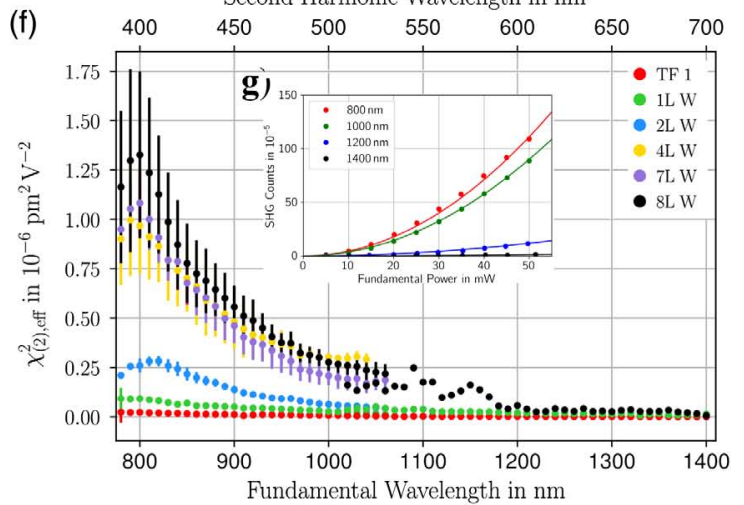

Figure 3. a,b,d,e) The SHG polarization responses for pump wavelengths of $800 \mathrm{~nm}$ (red dots), $1000 \mathrm{~nm}$ (green dots), $1200 \mathrm{~nm}$ (blue dots), and $1400 \mathrm{~nm}$ (black dots). The legends next to the polar plots describe by which value the signals were multiplied for better readability. c) SEM image of a one-layer woodpile structure. The corresponding SHG polarization response is placed on top of the image (red dots). The long axis of the dipole is exactly perpendicular to the orientation of the rods. The SHG polarization response was measured at a fundamental wavelength of $1450 \mathrm{~nm}$. f) Squared effective second-order susceptibility of one to eight layers of a woodpile photonic crystal compared with a normal unpatterned thin film. The measurements were taken in two different laser ranges (780-1060 nm [laser range] and 1060-1400 nm [OPO range]). g) SHG power dependence on the incident fundamental power for an eight-layer sample showing its quadratic behavior and, therefore, proves that our signals are indeed second harmonic ones.

the order of $1.0 \mathrm{~cm}^{2}$. To the best of our knowledge, this is the largest 3D PhC out of a nonlinear material produced so far. The linear optical characterization shows a transmission stop band at $757 \mathrm{~nm}$ with a relative linear transmission of $48.4 \%$ for a four-layer sample. The linear bandgap could experimentally not be seen because the bandgap only starts to build up after four layers, but the appearance of the side band gives evidence of coherent wave propagation and interference due to the woodpile PhC structure. Furthermore, we showed that there is a cubic PhC structure in the linear and nonlinear optical regime. The very good agreement between experiments and simulations gives an insight that controllable bottom-up realization of a full bandgap 3D NPhC is feasible in the future. By removing the defects to avoid scattering, this fabrication method can reach much higher performing structures, and by fabricating more layers, the bandgap will become visible in the future.

Our fabrication method of PhCs out of a nonlinear material provides an inexpensive alternative to common top-down fabrication approaches. Our structures can be integrated on any kind of optical components and materials. This will potentially enable large-scale fabrication of 3D NPhCs. By exploiting $\mathrm{BaTiO}_{3}$ 's strong electro-optical, elasto-optical, and thermo-optical properties, active 3D NPhCs could also be realized. ${ }^{[35-38]}$ 
In the future, we will focus on the controllability of the alignment between the layers and on the improvement of the surface roughness of the structures to achieve sharper bandgap structures and enhance efficiencies. Furthermore, the effective refractive index of the composite has to be increased to facilitate applications relying on a large effective index contrast. By replacing the sol-gel precursor with another polymer, we will be able to measure the nonlinear photonic bandgap in the future. The optimization of these structures will be on the material components as well as on the physical measurements.

\section{Experimental Section}

Materials: $\mathrm{BaTiO}_{3}$ NP dispersion in water $(25 \mathrm{wt} \%$ BT80 from Nyacol Nano Technology Inc., tetragonal phase, $50 \mathrm{~nm}$ diameter), Norland Optical Adhesive (NOA60, 30-60\% Norland Products Inc.), propylene glycol monomethyl ether acetate (PGMEA, ReagentPlus 99.5\%, Sigma-Aldrich), sol-gel (titanium-diisoproxide-bis (2,4-pentanedionate, $\mathrm{C}_{16} \mathrm{H}_{28} \mathrm{O}_{6} \mathrm{Ti}, 75 \mathrm{wt} \%$ in isopropanol, Gelest Inc.), 1-methyl-2-pyrrolidinone (NMP, ACS reagent $99.0 \%$ Sigma-Aldrich), methanol (MeOH, p.a. $99.9 \%$, Sigma-Aldrich), (heptadecafluoro-1,1,2,2-tetrahydrodecyl)dimethylchlorosilane $\left(\mathrm{C}_{14} \mathrm{H}_{16} \mathrm{~F}_{17} \mathrm{NSi}, 95 \%\right.$, Gelest Inc.), PDMS (Sylgard 184 silicone elastomer kit, Dowsil).

SEM and FIB: Figure $3 \mathrm{~d}$ and $\mathrm{S}$, Supporting Information, were taken with a FEI Magellan 400 SEM. Figure $1 \mathrm{~b}$ was taken with a FEI Quanta 200F SEM and, for Figure 1c, a Zeiss NVision 40 FIB-SEM was used. A metallic film could not be deposited for better visibility on the samples without destroying the optical signals and making the individual NPs invisible.

Linear Optical Measurements: The diffraction pattern images were taken from a screen directly after the sample with a single-lens reflex camera (Sony Alpha 77II).

We illuminated the samples by a broadband halogen fiber optic illuminator (OSL2 Fiber Collimator from Thorlabs). The light was collimated by several lenses and the polarization is controlled with a film polarizer (LPNIRE100-B from Thorlabs). The spot size of the incident light was controlled with an aperture of a $3 \mathrm{~mm}$ diameter. The light was collected with a fiber collimator (F280APC-1550 from Thorlabs) and was transmitted through a multimode fiber from AFW Technologies Inc. (core size $105 \mu \mathrm{m}, \mathrm{MM1}$-FC-FC-105/125-C-3-0.12NA). The analysis was performed with a high-precision spectrometer at a temperature of $-70^{\circ} \mathrm{C}$ (Acton SP-2356 from Princeton Instruments, equipped with a thermoelectrically cooled CCD camera, Pixis 256E). We used a grating with $150 \mathrm{~g} \mathrm{~mm}^{-1}$ and a blaze of 500 . The slit was open to $1 \mu \mathrm{m}$. We normalized the intensity of the sample by the signal measured through a blank glass substrate of the same thickness as the substrate using the formula $T=\left(I_{\text {Sample }}-I_{\text {Background }}\right) /\left(I_{\text {Substrate }}-I_{\text {Background }}\right)$. Afterward, the data were smoothened by a Savitzky-Golay filter, which was based on the principle of least squares. ${ }^{39]}$

The diffraction pattern of a four-layer woodpile was excited with a SLED (EXS210060-01 from Exalos, center wavelength $789.9 \mathrm{~nm}$ ) and the image was taken with a single-lens reflex camera (Sony Alpha 77II, exposure $15 \mathrm{~s}$ ).

Linear FDTD Simulation: Simulations were performed with Lumerical FDTD solutions (version 8.18.1332). The woodpile structure package from Lumerical was used. The woodpile was placed on a glass substrate surrounded by air. A mesh size of $0.0095 \mu \mathrm{m}$ was used for $x, y$, and $z$ direction. A frequency-domain field and power monitor were used to collect the signal for a wavelength range of $0.35-1.05 \mu \mathrm{m}$. The source was a plane wave of type Block/periodic. The structure parameters were pitch of $1.002 \mu \mathrm{m}$, height of $260 \mathrm{~nm}$, width of $506 \mathrm{~nm}$, and a refractive index of 1.775 .

Nonlinear Optical Measurements: Nonlinear optical measurement of the SHG spectra were measured with a nonscanning transmission optical microscope. ${ }^{[28]}$ We excited the samples with a tunable Ti:sapphire laser from coherent $(780-1060 \mathrm{~nm}$ with steps of $10 \mathrm{~nm})$ and an OPO Chameleon from APE (1060-1450 nm with steps of $10 \mathrm{~nm})$. The laser light was focused with a lens with a NA of 0.26 (A220TM from Thorlabs). The signal was collected with a $100 \times$ objective (NA $=0.8, L M P l a n F L ~ N$ from Olympus) and focused on a sCMOS camera (Zyla 4.2 from Andor). The fundamental wavelength was removed with two BG39 filters for the laser range and two FESH0900 filters from Thorlabs for the OPO range. We used exciting power values between 50 and $100 \mathrm{~mW}$ and typical exposure times of $500 \mathrm{~ms}$.

Further details of the crystal structure investigations may be obtained from the Fachinformationszentrum Karlsruhe, 76344 EggensteinLeopoldshafen (Germany), on quoting the depository number CSD67518 for cubic $\mathrm{BaTiO}_{3}$ and CSD-67520 for tetragonal $\mathrm{BaTiO}_{3}$.

\section{Supporting Information}

Supporting Information is available from the Wiley Online Library or from the author.

\section{Acknowledgements}

The authors acknowledge support from the Scientific Center of Optical and Electron Microscopy (ScopeM) of the Swiss Federal Institute of Technology (ETHZ), especially Dr. Karsten Kunze for taking the SEM image in Figure 1c. Furthermore, the authors acknowledge support from the operation team of the Binning and Rohrer Nanotechnology Center (BRNC) and the operation team of FIRST-Center for Micro and Nanoscience at ETHZ. Especially, Ute Drechsler gave a lot of helpful advice. The authors thank Dr. Frank Lichtenberg in the group of Prof. Spaldin in the Material Science Department of ETHZ for letting us use their high-temperature oven. The authors thank Damian Bucher for drawing Figure 1a. The authors thank Prof. Pratsinis of the Mechanical Engineering Department of ETHZ for letting us perform powder XRD measurements. The authors thank Annina Moser of the group of Prof. Wood at the Electrical Engineering Department of ETHZ for support with the photospectrometer measurements of NOA60 and pure $\mathrm{TiO}_{2}$ matrix thin films. The authors thank Prof. Ensslin from the Physics Department of ETHZ for letting us use his AFM. The authors thank Dr. Anton Sergeyev for the FIB cut of the sample in Figure 1d. The authors thank the Swiss National Science Foundation (SNF) grants 150609 and the Ambizione grant 179966, as well as the NCCR ETH FAST grant. This project has received funding from the European Union's Horizon 2020 research and innovation program/from the European Research Council under the Grant Agreement No. 714837 (Chi2-nano-oxides). The project has received funding from the European Union's Horizon 2020 research and innovation program under the Marie Skłodowska-Curie grant agreement No. 800487.

\section{Conflict of Interest}

The authors declare no conflict of interest.

\section{Keywords}

barium titanate, nonlinear optics, second harmonic generation, woodpiles, 3D photonic crystals

Received: December 9, 2019

Revised: January 22, 2020

Published online: February 16, 2020

[1] P. J. Pauzauskie, P. Yang, Mater. Today 2006, 9, 36.

[2] J. D. Joannopoulos, S. G. Johnson, J. N. Winn, R. D. Meade, Photonic Crystals - Molding the Flow of Light, Princeton University Press, Princeton 2007. 
[3] R. W. Boyd, Nonlinear Optics, 2nd ed., Academic Press, San Diego, CA 2003, pp. 533-559.

[4] S. Mingaleev, Y. Kivshar, Opt. Photonics News 2002, 13, 48.

[5] T. Xu, D. Lu, H. Yu, H. Zhang, Y. Zhang, J. Wang, Appl. Phys. Lett. 2016, 108, 51907.

[6] T. Xu, K. Switkowski, X. Chen, S. Liu, K. Koynov, H. Yu, H. Zhang, J. Wang, Y. Sheng, W. Krolikowski, Nat. Photonics 2018, 12, 591.

[7] K.-H. Chang, J.-Y. Han, L.-H. Peng, A. Boudrioua, in High-Brightness Sources and Light-Driven Interactions Congress, OSA Technical Digest, Optical Society of America, Strasbourg, France 2018, p. JT5A.8.

[8] D. Wei, C. Wang, H. Wang, X. Hu, D. Wei, X. Fang, Y. Zhang, D. Wu, Y. Hu, J. Li, S. Zhu, M. Xiao, Nat. Photonics 2018, 12, 596.

[9] M. R. Escalé, D. Pohl, A. Sergeyev, R. Grange, Opt. Lett. 2018, 43, 1515.

[10] S. Diziain, R. Geiss, M. Zilk, F. Schrempel, E. Kley, A. Tünnermann, T. Pertsch, R. Geiss, M. Zilk, F. Schrempel, E. Kley, in 16th Int. Conf. on Transparent Optical Networks (ICTON), IEEE, Graz 2013.

[11] N. G. R. Broderick, G. W. Ross, D. J. Richardson, D. C. Hanna, Nonlinear Guided Waves and Their Applications, Optical Society of America, Dijon 1999, p. PD1.

[12] D. Staedler, T. Magouroux, R. Hadji, C. Joulaud, J. Extermann, S. Schwung, S. Passemard, C. Kasparian, G. Clarke, M. Gerrmann, R. Le Dantec, Y. Mugnier, D. Rytz, D. Ciepielewski, C. Galez, S. Gerber-Lemaire, L. Juillerat-Jeanneret, L. Bonacina, J.-P. Wolf, ACS Nano 2012, 6, 2542.

[13] K. M. Ho, C. T. Chan, C. M. Soukoulis, R. Biswas, M. Sigalas, Solid State Commun. 1994, 89, 413.

[14] S. Y. Lin, J. G. Fleming, D. L. Hetherington, B. K. Smith, R. Biswas, K. M. Ho, M. M. Sigalas, W. Zubrzycki, S. R. Kurtz, J. Bur, Nature 1998, 394, 251.

[15] S. Dhuey, A. Koshelev, N. Borys, J. R. Piper, M. Melli, J. P. Schuck, C. Peroz, S. Cabrini, J. Phys. Commun. 2017, 1, 15004.

[16] M. R. Beaulieu, N. R. Hendricks, J. J. Watkins, ACS Photonics 2014, 1, 799.

[17] M. Deubel, G. von Freymann, M. Wegener, S. Pereira, K. Busch, C. M. Soukoulis, Nat. Mater. 2004, 3, 444.

[18] R. Jiawook, B. Heidari, in Emerging Applications of Nanoparticles and Architectural Nanostructures: Current Prospects and Future Trends (Eds: A. S. H. Makhlouf, A. Barhoum), Elsevier, Amsterdam 2018, pp. 141-176.

[19] F. Timpu, A. Sergeyev, N. R. Hendricks, R. Grange, ACS Photonics 2017, 4, 76.

[20] M. Niederberger, N. Pinna, J. Polleux, M. Antonietti, Angew. Chem. 2004, 116, 2320.
[21] E. Kim, A. Steinbrück, M. T. Buscaglia, V. Buscaglia, T. Pertsch, R. Grange, ACS Nano 2013, 7, 5343.

[22] S. Khadir, P. Bon, D. Vignaud, E. Galopin, N. McEvoy, D. McCloskey, S. Monneret, G. Baffou, ACS Photonics 2017, 4, 3130.

[23] M. V. Rybin, K. B. Samusev, S. Y. Lukashenko, Y. S. Kivshar, M. F. Limonov, Sci. Rep. 2016, 6, 30773.

[24] Y. A. Vlasov, M. A. Kaliteevski, V. V. Nikolaev, Phys. Rev. B 1999, 60, 1555.

[25] A. F. Koenderink, W. L. Vos, Phys. Rev. Lett. 2003, 91, 213902.

[26] W. L. Vos, T. W. Tukker, A. P. Mosk, A. Lagendijk, W. L. IJzerman, Appl. Opt. 2013, 52, 2602.

[27] F. García-Santamaría, J. F. Galisteo-López, P. V. Braun, C. López, Phys. Rev. B 2005, 71, 195112.

[28] M. Timofeeva, L. Lang, F. Timpu, C. Renaut, A. Bouravleuv, I. Shtrom, G. Cirlin, R. Grange, Nano Lett. 2018, 18, 3695.

[29] G. Saerens, L. Lang, C. Renaut, F. Timpu, V. Vogler-Neuling, C. Durand, M. Tchernycheva, I. Shtrom, A. Bouravleuv, R. Grange, M. Timofeeva, Opt. Express 2019, 27, 19915.

[30] F. Timpu, M. Reig Escalé, M. Timofeeva, N. Strkalj, M. Trassin, M. Fiebig, R. Grange, Adv. Opt. Mater. 2019, 7, 1900936.

[31] S. K. Das, C. Schwanke, A. Pfuch, W. Seeber, M. Bock, G. Steinmeyer, T. Elsaesser, R. Grunwald, Opt. Express 2011, 19, 16985.

[32] C. Wong, Y. Y. Teng, J. Ashok, P. L. H. Varaprasad, Handbook of Optical Constants of Solids, Academic Press, Burlington 1997, pp. 789-803.

[33] J. L. P. Hughes, Y. Wang, J. E. Sipe, Phys. Rev. B 1997, 55, 13630.

[34] W. R. L. Lambrecht, S. N. Rashkeev, Phys. Status Solidi B 2000, 217, 599.

[35] S. Abel, T. Stöferle, C. Marchiori, C. Rossel, M. D. Rossell, R. Erni, D. Caimi, M. Sousa, A. Chelnokov, B. J. Offrein, J. Fompeyrine, Nat. Commun. 2013, 4, 1671.

[36] K. D. Fredrickson, V. V. Vogler-Neuling, K. J. Kormondy, D. Caimi, F. Eltes, M. Sousa, J. Fompeyrine, S. Abel, A. A. Demkov, Phys. Rev. B 2018, 98, 75136.

[37] S. Kroesen, K. Tekce, J. Imbrock, C. Denz, Appl. Phys. Lett. 2015, 107, 101109.

[38] S. Abel, F. Eltes, J. E. Ortmann, A. Messner, P. Castera, T. Wagner, D. Urbonas, A. Rosa, A. M. Gutierrez, D. Tulli, P. Ma, B. Baeuerle, A. Josten, W. Heni, D. Caimi, L. Czornomaz, A. A. Demkov, J. Leuthold, P. Sanchis, J. Fompeyrine, Nat. Mater. 2018, 18, 42.

[39] A. Savitzky, M. J. E. Golay, Anal. Chem. 1964, 36, 1627. 